\title{
Spinal hydatic cyst
}

\author{
Iuliana Madalina Cocianga ${ }^{1}$, Ana-Maria Enachi', Corina Manuela Constantin ${ }^{2}$, Vlad Nedelcu', \\ Cristina Baetu' ${ }^{1}$ Armand Daniel Frasineanu' ${ }^{1}$ Gabriela Mihailescu ${ }^{1,3}$ \\ ${ }^{1}$ Colentina Clinical Hospital, Neurology Department, Bucharest, Romania \\ ${ }^{2}$ Colentina Clinical Hospital, Parasitology Department, Bucharest, Romania \\ ${ }^{3}$ University of Medicine and Pharmacy Carol Davila, Bucharest, Romania
}

ABSTRACT
Spinal hydatic disease must be considered in the differential diagnosis of the spinal cord compression syndrome
especially in endemic areas. The disease is highly prone to recurrence, despite the introduction of modern surgical
and pharmacological therapies. The prognosis is poor, comparable to that of malignancies.
Keywords: spinal hydatic cyst, imaging, spinal cord compression, back pain

\section{INTRODUCTION}

Cystic echinococcosis (CE) is a zoonosis caused by the larval stage of the tapeworm Echinococcus granulosus. In humans, the infection induces the formation of parasitic cyst mostly in the liver and lungs, but virtually any organ can be affected. CE of the bone is one of the rarest forms of the disease, yet it is also extremely debilitating for patients and hard to manage for clinicians [1]. Only 0.5 to $3.1 \%$ of patients suffer from bone involvement, half of which occur in the spine [2]. This localization marks the torpid, insidious progression of the parasite into the bone tissue, leading to an immediate diffuse, extensive invasion process [3].

The usual complaints are a long history of back pain and/or signs of spinal cord compression. Cauda equina syndrome, paraparesis or paraplegia were also reported. Rupture of the cyst with spillage of the content may generate a variety of hypersensitivity reactions, even anaphylactic shock $[4,5]$.

Surgery is the treatment of choice for spinal echinococcosis, following two primary tasks: the decompression of the compromised spinal cord and the stabilization of a compromised spinal column [4].

\section{CASE REPORT}

We present the case of a 67-year-old man, known with four surgical interventions for pulmonary hydatic cyst performed in 1990, 1994, 1997 and the last one in December 2017. In 2003 he was treated with albendazole $400 \mathrm{mg}$ twice daily, but due to personal reasons, he stopped the treatment and restarted it after the last surgical intervention. He came in the neurology department complaining of left hand weakness and low cervical pain. The symptoms started insidiously two months before the admission and the neurological examination.

The neurological examination revealed: low cervical pain radiating to the medial part of the left arm and forearm, finger 4 and 5 of the left hand paresthesias (numbness) and weakness for the hand grip.

He performed an cervical-thoracic spinal MRI, with the following pathological findings: An osteolytic, multiloculate lesion, with fluid signal (cystic 
lesion), with epidural and left paravertebral extension and with compression of the spinal cord (Figure 1,2$)$ localized at the level of the first thoracic vertebra; the same kind of lesions are seen in the $\mathrm{C} 7$ and $\mathrm{T} 2$ vertebrae (Figure 3 ). In the proximity of left paravertebral extended lesion there is a cystic apical left pulmonary lesion (Figure 4). In the T1 MRI images the cysts appear hypo intense and the cyst walls enhance slightly after Gadolinium injection. In the $\mathrm{T} 2$ images, cysts are hyper intense, with a hypo intense cystic wall.

Taking into account the past medical history of the patient and the specific MRI images, the lesions are highly suggestive for spinal hydatic cysts.

\section{DISCUSSION}

The differential diagnosis should be made with: tuberculous spondylitis also known as Pott disease (important perilesional edema and important destruction of the vertebrae), vertebral pyogenic infection (osteomyelitis), spinal tumors: giant cell tumor, plasmocitoma, enchondroma (the lesions are not so well delimited and there is invasion of the soft tissue), aneurismal bone cyst. The fact that the lesions are cystic, multiloculate, with paravertebral extension, present in the overlying and underlying vertebra and there is no important bone destruction it stands for the diagnosis of spinal hydatic cyst [6].

The patient should perform a surgical intervention. Simple decompression with laminectomy is the most commonly reported procedure. Bone involvement is a major challenge for the treating surgeon. Even laminectomy may result in rupture of the cyst and spillage [3].

There are a lot serious surgical complications like perioperative death, recurrence, increasing neurological deficits, secondary infection, cerebrospinal fluid fistula and anaphylaxis. Up to $40 \%$ of patients are reported to have recurrent symptomatology within 2 years following the initial surgical intervention $[2,3]$.

Pharmacotherapy with Albendazole (800 mg daily in two divided doses) should be continued indefinitely.

Despite the aggressive treatment, there is a poor survival, considered as only 5 years after the symptom onset [2].

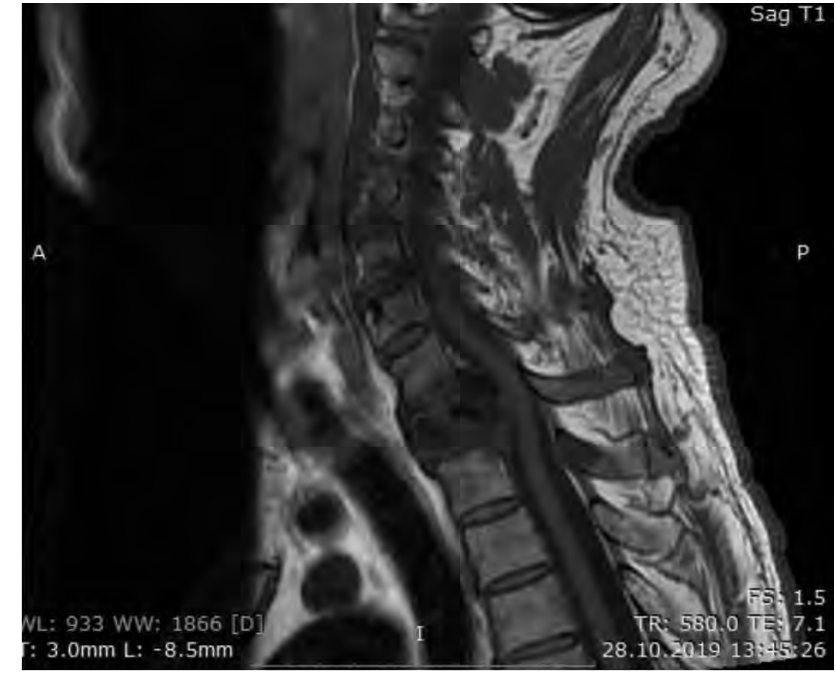

FIGURE 1. Sagittal T1 MRI - hypo intense, osteolytic, multiloculate lesion with fluid signal a the level of the T1 vertebra

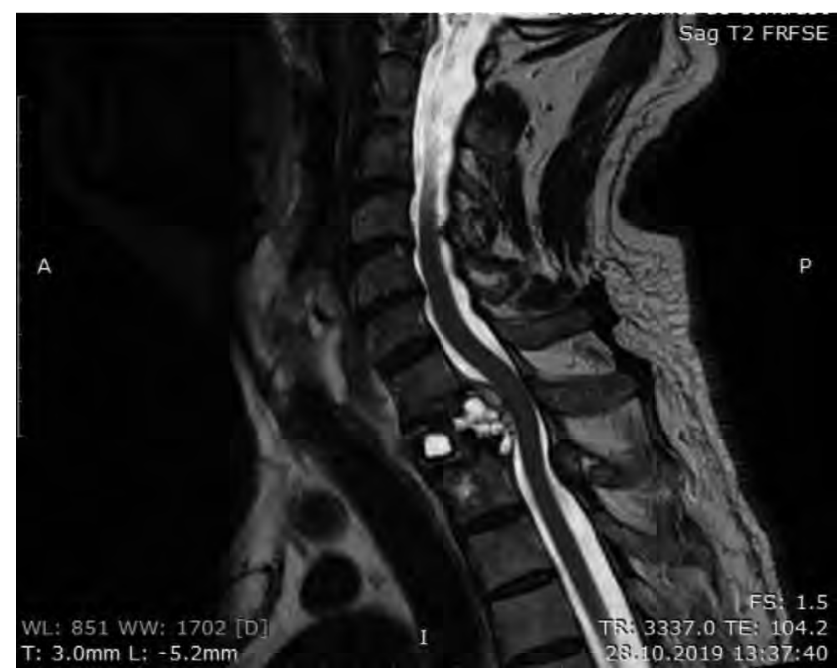

FIGURE 2. Sagittal T2 MRI - hyper intense osteolytic, multiloculate lesion with fluid signal

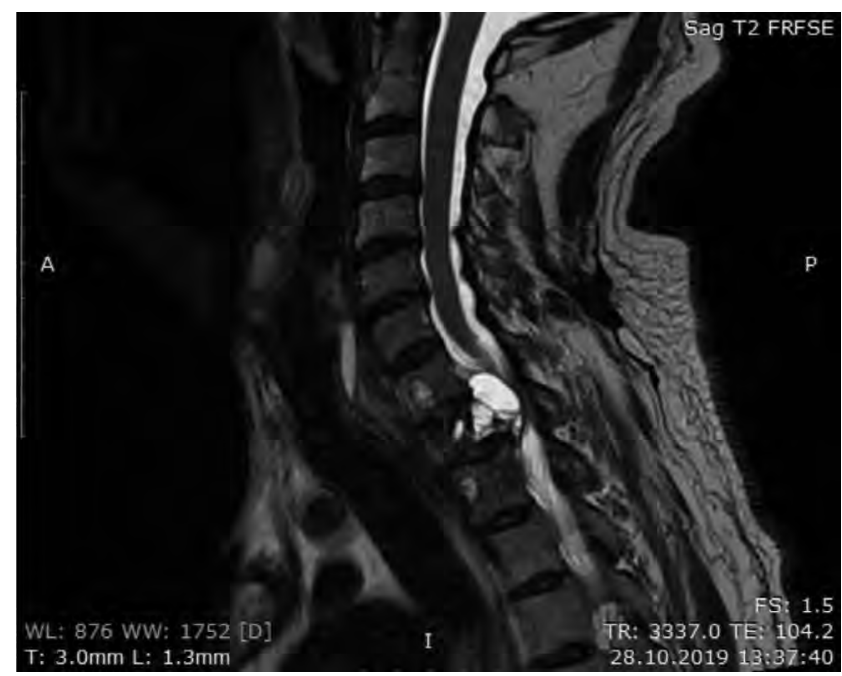

FIGURE 3. Sagittal T2 MRI - similar cystic lesions at the level of the $C 7$ and $T 1$ vertebra 


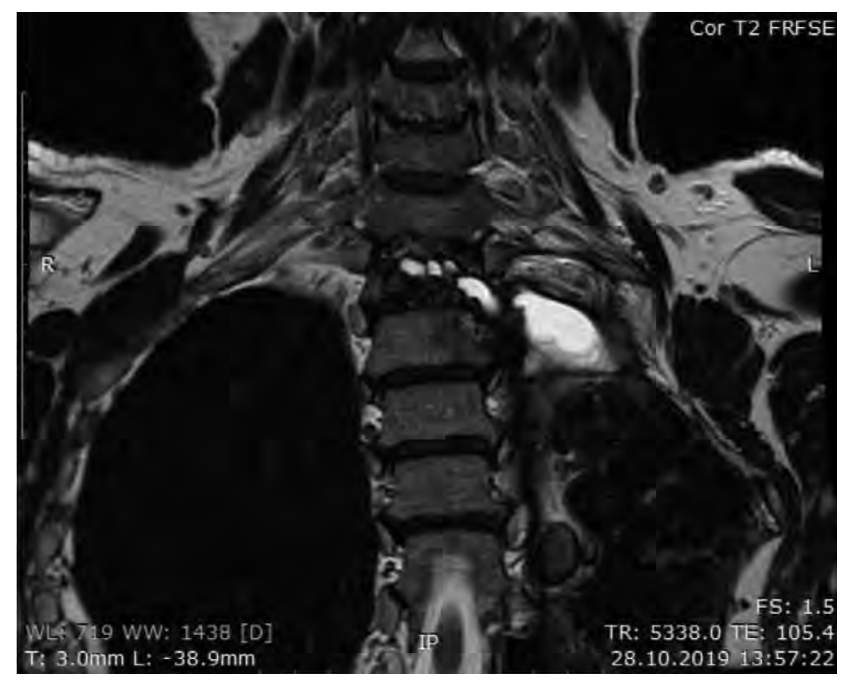

FIGURE 4. Axial T2 MRI - hyper intense left cystic apical pulmonary lesion in the proximity of the paravertebral extended lesion

\section{REFERENCES}

1. Cattaneo L, Manciulli T, Cretu CM et al. Cystic Echinococcosis of the Bone: A European Multicenter Study. Am J Trop Med Hyg. 2019;100(3):617.

2. Pamir MN, Ozduman K, Elmaci I. Spinal hydatic disease. Spinal Cord. 2002;40:153-160.

3. Zlitni M, Ezzaouia K, Lebib H et al. Hydatic Cyst of bone: Diagnosis and treatment. World J Surg. 2001;25(1):75.

4. www.emedicine.medscape.com - Echinococcosis hydatic cyst treatment and management,

5. www.uptodate.com - Clinical manifestation and diagnosis of echinococcosis,

6. www.radiopaedia.org - Spinal hydatic disease,

Conflict of interest: none declared Financial support: none declared 